\title{
Effects of gonadotrophin deficiency on follicular development in hypogonadal (hpg) mice
}

\author{
D. M. G. Halpin†, H. M. Charlton and M. J. Faddy* \\ Department of Human Anatomy, University of Oxford, South Parks Road, Oxford OXI 3QX, U.K. \\ and $^{*}$ Department of Statistics, University of Birmingham, Birmingham B15 2TT, U.K.
}

\begin{abstract}
Summary. The rates of follicle growth and death in GnRH-deficient hypogonadal (hpg) mice and in normal mice were studied using the stochastic compartmental model of follicle dynamics. The rate estimates derived from this model suggest that in normal mice gonadotrophins act at several stages in the development of ovarian follicles. Gonadotrophins appear to regulate the number of follicles beginning to grow by controlling both the rate at which non-growing follicles enter the growing pool and the loss of non-growing follicles to atresia. They also appear to promote the growth of mediumsized follicles by reducing the rate of loss to atresia of these follicles rather than by stimulating growth per se. Furthermore, these data suggest that an intra-ovarian autoregulatory mechanism may exist to control the number of large follicles that are formed.
\end{abstract}

\section{Introduction}

Ovarian follicle development is known to be an hormonally dependent process, although the initial trigger for primordial follicle growth and the developmental stages at which gonadotrophic hormones act remain unclear. The effects on the follicle population of both age and hypophysectomy have been studied in quantitative analyses in rats and mice (Arai, 1920; Zuckerman, 1951; Mandl \& Zuckerman, 1952; Jones \& Krohn, 1961a, b). The kinetics of follicle development have been studied using the pulse-labelling technique (Pedersen, 1969, 1972; Pedersen \& Peters, 1971), but this technique fails to identify the role played by atresia.

An alternative approach to the study of the kinetics of follicular growth is the mathematical method by which static datum points obtained from differential follicle counts are used to estimate rates of migration (growth) and death (atresia) (Faddy, 1976; Faddy, Jones \& Edwards, 1976). We have used this method to study the kinetics of follicular growth in GnRH-deficient hypogonadal (gene symbol hpg) mice, which as a consequence of this deficiency have a marked reduction in pituitary and plasma gonadotrophin concentrations, with severe hypogonadism (Cattanach, Iddon, Charlton, Chiappa \& Fink, 1977). In the ovaries of hpg females the number of growing follicles of all sizes is significantly reduced and follicular growth does not proceed beyond the preantral stage (Halpin, Jones, Fink \& Charlton, 1986). A comparison of follicular transition and death rates in mutants and their normal littermates has therefore been used to identify the stages at which gonadotrophins regulate follicle development.

\section{Materials and Methods}

The mice used in these studies were hpg mutants or normal animals reared in the Department of

$\dagger$ Present address: Department of Obstetrics and Gynaecology, St Thomas's Hospital Medical School, London SE1 7EH, U.K. 
Human Anatomy, Oxford, from an original stock provided by the MRC Radiobiology Unit, Harwell. Females were weaned at 18-20 days of age and maintained as described by Halpin $e t$ al. (1986).

The day of birth was designated as Day 0 and groups of 5-10 virgin hpg and normal females were killed at various ages from 7 to 120 days. In line with other studies (e.g. Jones \& Krohn, 1961 b) it was considered more important to kill the normal animals at an exact age rather than on a specific day of the oestrous cycle. The ovaries were removed, embedded in paraffin wax and sectioned serially at $7 \mu \mathrm{m}$. The sections were stained with haematoxylin and eosin, and for each animal the total number of follicles was counted using the classification scheme of Faddy et al. (1976) which is based upon that of Pedersen \& Peters (1968). Follicles were classified into one of 5 types based upon the number of layers of granulosa cells and the morphology of the follicle. Group I follicles correspond to types 1, 2 and 3a of Pedersen \& Peters's (1968) scheme and comprise oocytes surrounded by a single layer of less than 20 granulosa cells. Group II follicles correspond to type $3 \mathrm{~b}$ and have a single layer of more than 20 granulosa cells, whilst Group III have two layers of granulosa cells and correspond to type 4 follicles, and Group IV have three layers of granulosa cells and are equivalent to type 5a follicles. All follicles larger than this, with or without an antrum, are

(a)

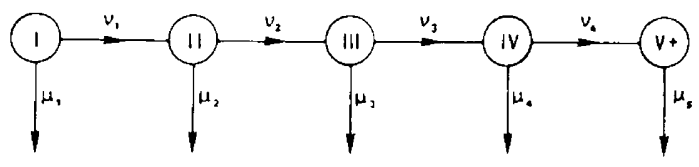

(b)

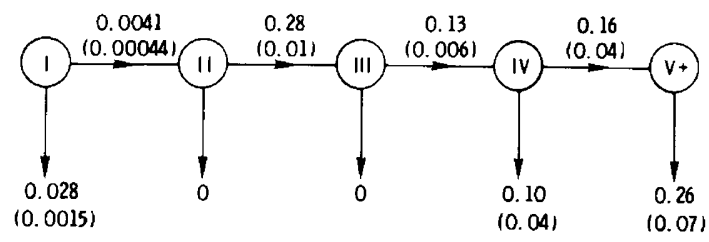

(c)

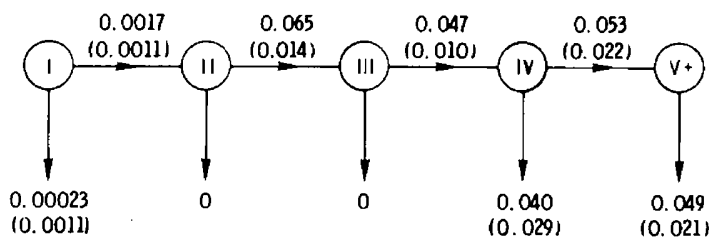

(d)

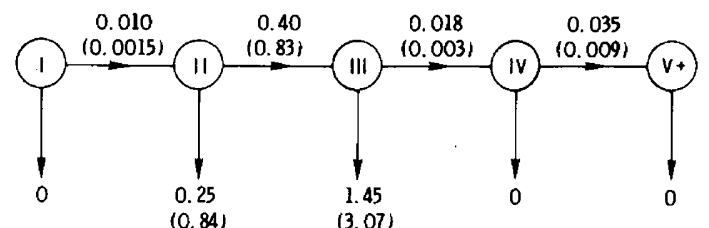

(e)

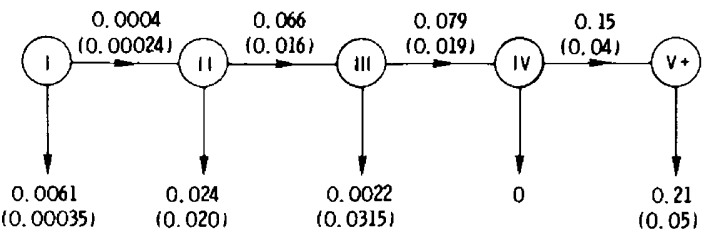

Fig. 1. Estimates of the growth $(v)$ and death rates $(\mu)$ and their corresponding standard errors (in parentheses) for each of the five stages of follicular development, Groups I-V +, in normal and hypogonadal mice: (a) the model; (b) normal mice before 30 days; (c) normal mice after 30 days; (d) hypogonadal mice before 30 days; (e) hypogonadal mice after 30 days. 
classified together as Group $\mathrm{V}+$, which therefore includes follicles of type $5 \mathrm{~b}, 6,7$ and 8 of Pedersen \& Peters's (1968) scheme.

The data were analysed using the compartmental model for ovarian follicle dynamics (Faddy, 1976; Faddy et al., 1976), which describes the growth of the follicles through five stages (Groups $\mathrm{I}-\mathrm{V}+$ ) by five corresponding compartments (Fig. 1). In essence, this model assumes that movement occurs out of Group I and into Group II and subsequent Groups by migration so that Groups are interdependent in that, for example, the number of follicles in Group IV is influenced by those in the three preceding Groups, and in turn influences the number of follicles in Group $\mathrm{V}+$. It is also assumed that follicles may leave the Groups by death, and that the rates of migration $v_{1}(t)$ (from I to II), $v_{2}(\mathrm{t})$ (from II to III), $v_{3}(\mathrm{t})$ (from III to IV) and $v_{4}(\mathrm{t})$ (from IV to $\mathrm{V}+$ ), and death $\mu_{1}(\mathrm{t})$ (from I), $\mu_{2}(\mathrm{t})$ (from II), $\mu_{3}(\mathrm{t})$ (from III), $\mu_{4}(\mathrm{t})$ (from IV) and $\mu_{5}(\mathrm{t})$ (indicating egress as a result of death and ovulation from $\mathrm{V}+$ ) are all age dependent. Such a mathematical model can be formulated stochastically, as in Faddy (1976), with mean Group sizes $\left(\lambda_{i}(t), i=1,2, \ldots, 5\right)$ given by the solution (see Faddy et al., 1976) of the system of differential equations:

$$
\frac{d \lambda_{i}(t)}{d t}=v_{i-1}(t) \lambda_{i-1}(t)-\left[v_{i}(t)+\mu_{i}(t)\right] \lambda_{i}(t),
$$

with $v_{0}(t)$ and $v_{5}(t)$ both 0 , since migration into Group I and out of Group V+ is not permitted. The model thus describes the process of follicular maturation throughout life in terms of the numbers of growing follicles in each of these five stages and curves showing the mean number of follicles at each of these stages at any age can be calculated from the estimated migration and death rates.

Since rates of migration and death of follicles in previous studies had been found not to be constant throughout life, a simple form of age dependence was used in which two phases, designated phase 1 (immature) and phase 2 (mature) were identified and rates within each phase were assumed to be constant. Such age-dependence results in biphasic curves for the mean numbers of follicles, and the appropriateness of the resulting model was assessed by comparing its fit with that of a single-phase model. The transition between these phases was taken to be 30 days of age: the mean time at which vaginal opening occurred in the normal mice, and co-incidentally the time of an apparent trough in the Group II counts. The migration and death rates and their approximate standard errors were estimated using the same techniques as in Faddy et al. (1976), and Faddy, Gosden \& Edwards (1983).

\section{Results}

The estimated rates of follicular migration and death together with their standard errors are shown in Fig. 1, and the concordance between the calculated profile of changes in the number of follicles with increasing age in both normal and $h p g$ mice and the observed changes are shown in Fig. 2. In normal mice the loss of Group I follicles was well described by the model, and the curves for follicles of Groups II to V+ corresponded well with the observed counts. A similar two-phase description of follicle growth in the mutants also corresponded well with the observed counts, showing a significant improvement over a single phase model $(P<0.001)$.

The estimated number of follicles present at Day 0 in normal mice was $6165 \pm 171$ and in mutants it was $4786 \pm 152$. The rapid loss of Group I follicles in normal animals during the first 30 days was largely accounted for by death, with about 7 times more follicles dying than growing, but from Day 30 onwards, death from Group I was negligible. No death from Groups II and III was detected in either phase, and the rates of growth of follicles from Group II to Group III and from Group III to Group IV were both significantly reduced after Day $30(P<0.001)$. Approximately equal numbers of follicles left Group IV by growth and death, both before and after Day 30 . Both 

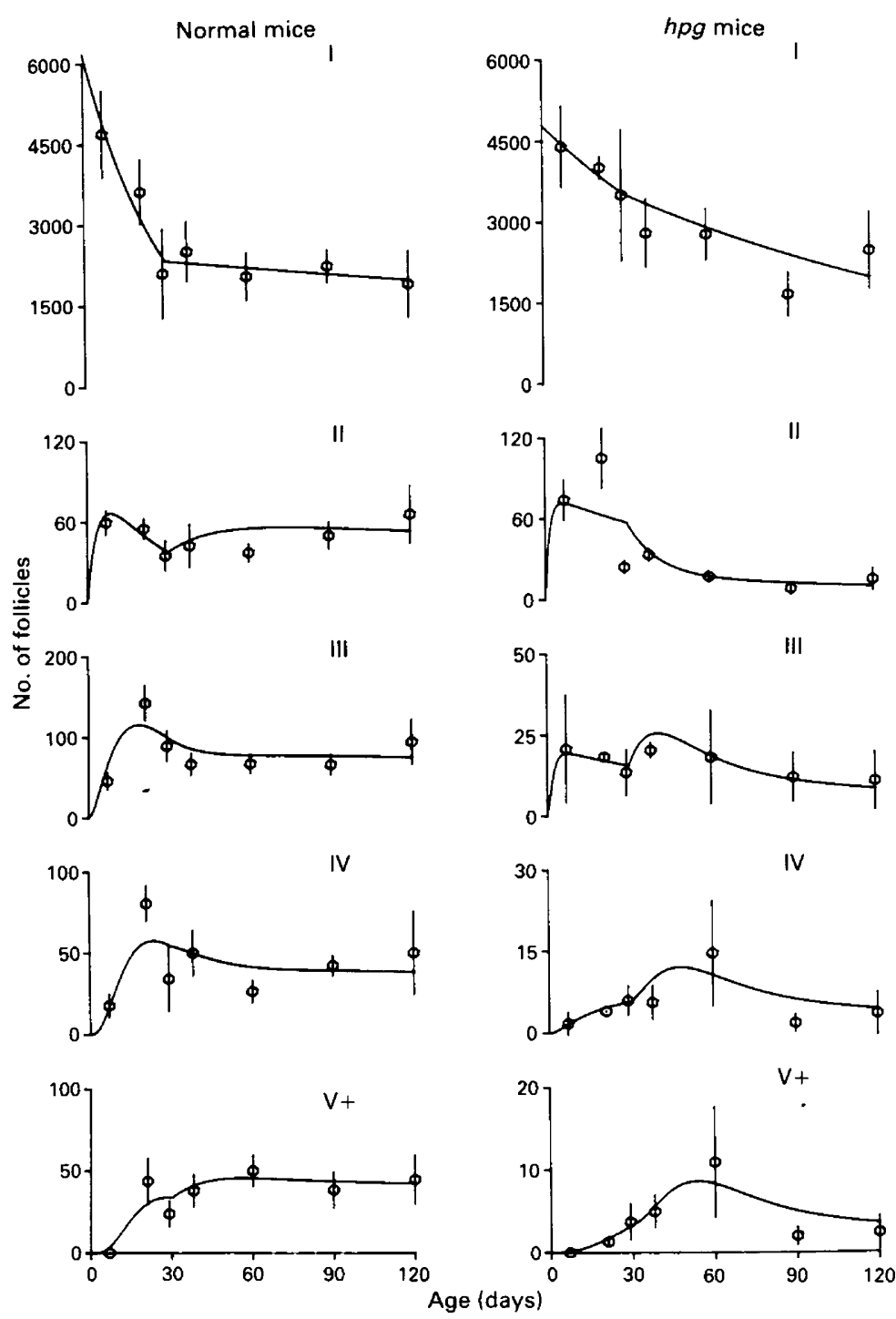

Fig. 2. Variation in the numbers of follicles of different sizes (Groups $I-V+$ ) in normal and hypogonadal $(\mathrm{hpg})$ mice from birth to 120 days of age. The results of differential follicle counts are shown as the mean \pm standard deviation and the continuous lines show the estimated mean predicted from the 'death-migration model' (Faddy et al., 1976, 1983).

rates were reduced during the second phase, but only the reduction in the growth rate was significant $(P<0.01)$. A significant reduction in the egress rate from Group $\mathrm{V}+$ was also observed in the second phase.

In mutant animals the estimates indicate that all follicles leaving Group I before Day 30 did so by growing, at a rate about double that in normal mice. After Day 30, the growth rate from Group I was significantly reduced in $h p g$ females $(P<0.001)$, whilst the death rate was increased $(P<0.001)$, with the result that many more follicles were lost by death than by growth, unlike normal mice in which negligible death from Group I occurred after 30 days. The estimated death rates 
from Groups II and III were accompanied by large standard errors; however, the poor fit of a model in which the death rates for both Group II and Group III were set to zero showed that significant death was occurring from one or other of these Groups $(P<0.001)$, but it was not possible to identify precisely which. Nevertheless, it is clear that relatively few follicles progress beyond Group III. There was no death from Group IV at any time, or from Group V+ before 30 days.

During the second phase, the loss of follicles from Group I by death was significantly greater in $h p g$ than in normal animals $(P<0.001)$, whereas the rate of growth was lower, but not significantly, before Day 30, the growth rate from Group III was about 7 times lower in mutants than in normals, and the growth rate from Group IV was about four times lower in mutants. However, after Day 30, these growth rates were significantly increased $(P<0.001)$, whereas in normal animals a reduction was observed. The growth rates from Groups II and III were comparable in normal and mutant mice from Day 30 onwards. However, death of larger follicles occurred at a rate significantly higher than normal in mutants $(P=0.013)$, and this was also higher than the value, before 30 days, indicating that those few follicles that reach this stage die rapidly.

\section{Discussion}

The stochastic compartmental model has been used previously to estimate the kinetics of follicle development in various strains of mice, and significant differences have been observed between strains (Faddy et al., 1976, 1983). However, the physiological mechanisms underlying these differences are not known. Hypogonadal mice have a specific deficiency of GnRH with a consequent reduction in pituitary and plasma gonadotrophin concentrations, and $h p g$ females are therefore excellent for examination of the effects of markedly reduced gonadotrophic stimulation on the kinetics of follicle development.

The hpg mutation is on a house mouse background, derived originally from $\mathrm{F}_{1}$ hybrids of two inbred strains, $\mathrm{C} 3 \mathrm{H} / \mathrm{HEH}$ and $101 \mathrm{H}$, and the normals used in these studies had the same genetic background. The estimated total number of follicles present at birth in the normal animals was significantly lower than the number reported in other strains (Faddy et al., 1983), and the total number of follicles in hpg mice was estimated to be significantly lower than in normals. Genetic variation in the total number of ovarian follicles is well recognized, but the factors responsible for these differences are not known. It may be that the normal development of the hypothalamic/ pituitary axis results in prenatal secretion of gonadotrophic hormones which may regulate the total number of follicles; however, there is no evidence for this at present.

The estimated growth and death rates in the normal animals were of the same order of magnitude as those observed in studies of other strains of mice (Faddy et al., 1983). As in these studies, the majority of follicles leaving Group I in normal animals in the prepubertal period did so by dying; however, in the mutants, no death from Group I occurred during this phase, suggesting that gonadotrophins may have a role in the regulation of this process. It has previously been argued that lack of trophic stimulation is unlikely to account for the high death rate seen in normal animals during the prepubertal phase (Faddy et al., 1983), and the present data suggest that low levels of gonadotrophins do not result in increased death of Group I follicles. In fact, plasma FSH concentrations are high during the first 10 days of life in normal mice but are undetectable in $h p g$ mice during this period (Halpin et al., 1986). High concentrations of gonadotrophins may, therefore, inhibit growth from Group I at this time, perhaps as a result of down regulation of follicle FSH receptors, leading to an increase in death from this group.

In the post-pubertal period, the growth of follicles from Group I in hpg mice was reduced whilst the death rate was increased, and when compared with the equivalent rates in normal mice it seems that deficient gonadotrophic stimulation may lead to decreased initiation of follicle growth in this period, with a consequent increase in the rate of atresia of non-growing follicles. These data support the view that gonadotrophins do have a role in the initiation of follicle growth (Halpin et al., 
1986) and they appear to do this by regulating the rate at which small, non-growing follicles enter the growing pool, as well as reducing the rate at which non-growing follicles are lost by atresia.

Perhaps surprisingly, the growth and death rates in mutants were different in the prepubertal and post-pubertal periods. This may have been due to peri-pubertal changes in ovarian stimulation by other hormones, such as thyroid hormones or growth hormone, or it may be the result of a fundamental change in the intra-ovarian regulation of follicle development. There is now considerable evidence that many of the endocrine changes of puberty may be initiated by the ovary and that hypothalamic-pituitary changes are secondary to this process (Ojeda, Andrews, Advis \& Smith White, 1980). The changes in the growth and death rates of follicles in hpg mice may therefore be the result of an initial intra-ovarian change, which is not sustained by an increase in gonadotrophic hormone stimulation.

Gonadotrophic stimulation appeared to have a marked effect on the development of medium sized follicles, since in mutants death from Groups II and III was detected, whereas in normals there was no death at this stage. This agrees well with the observation that, in mutants, many Group III follicles appear to undergo atresia (Halpin, 1983). Gonadotrophins therefore appear to stimulate the growth of medium-sized follicles by reducing the rate of atresia, rather than by increasing the rate of growth per se. This effect has not previously been described, and the mechanism by which the rate of atresia is reduced is unknown; however, it may be an important action of gonadotrophins.

Because of the structure of the model, it was not possible to examine the effects of the $h p g$ mutation on the development of antral follicles; however, descriptive studies have shown that follicle development does not proceed beyond the pre-antral stage in hpg females (Halpin et al., 1986). The increased number of type $\mathrm{V}+$ follicles present in normal mice in the post-pubertal period also appears to be due to decreased death from Groups IV and V+ rather than increased growth rate, and this is in agreement with the findings of Hirshfield \& Midgley (1978), who suggested that FSH stimulates the production of antral follicles by rescuing them from atresia. In $h p g$ females, the rate of growth of follicles from Groups III and IV was significantly higher during the second phase than during the first phase; whereas in normal mice a reduction in the rate of growth of large follicles was observed during the second phase. Thus, although the number of follicles reaching Group III is markedly reduced in hpg females, those follicles that do reach this stage appear to be stimulated to grow at an increased rate. The same phenomenon is seen in normal animals during the pre-pubertal phase. It is possible, therefore, that once sufficient antral follicles have been formed, an autoregulatory mechanism exerts a negative feedback effect upon the growth of medium-sized follicles, inhibiting their development. This mechanism may be similar to the inhibitory effects of ovulatory follicles in large animals (diZerega \& Hodgen, 1980), and may explain the remarkable constancy of the numbers of large follicles despite considerable variation in the number of small follicles.

In some respects, the kinetics of follicle growth in hpg mice was similar to that after hypophysectomy. In hpg and hypophysectomized normal mice, substantial death of medium-sized follicles was observed, with few follicles progressing beyond Group III (Faddy et al., 1976, 1983). Growth from Group II through to Group IV occurred at a similar rate in hpg as in normal females in the mature phase. This is in agreement with the work of Nakano, Mizumo, Katayama \& Tojo (1975), who found that growth of medium-sized follicles occurred at similar rates in hypophysectomized and intact rats. Hypophysectomy also led to rapid death in Group V + of the few follicles progressing beyond Group III (Faddy et al., 1976, 1983), similar to that seen in hpg females, and this agrees with many previous studies showing that gonadotrophins are required for the formation of antral follicles (Richards, 1980). However, there were some important differences between the effects of hypophysectomy and the hypogonadal mutation in the mature phase. Death of follicles from Group I was reduced by hypophysectomy, whereas in $h p g$ mice the rate of death from this group was increased compared with normals. Furthermore, growth from Group I was markedly reduced in $h p g$ mice but not in hypophysectomized animals. The explanation for these differences is not 
clear, but it may be that there is a specific deficiency of gonadotrophins in hpg mice which has been present since birth, whereas in hypophysectomized animals the secretion of all pituitary hormones is abolished and, before hypophysectomy, the ovary had been subject to normal gonadotrophic stimulation.

An analysis of the kinetics of follicle growth in hypogonadal and normal mice using the stochastic compartmental model therefore provides information about the effects of follicle growth of a congenital lack of gonadotrophic stimulation, thereby indicating the ways in which gonadotrophins regulate follicle growth in normal animals. Previous studies have provided evidence both for and against an effect of gonadotrophins on the initiation of follicle growth (Peters, Byskov, Lintern-Moore, Faber \& Anderson, 1973; Lintern-Moore, 1977); however, the present studies clearly indicate that, in the absence of gonadotrophins, fewer Group I (non-growing) follicles are stimulated to grow, and more of these follicles are lost to atresia. These studies also suggest that gonadotrophins act at later stages during the growth of a follicle, facilitating the growth of medium-sized follicles by reducing the rate of atresia, and stimulating the growth of large follicles, again by rescuing them from atresia. Moreover, they provide evidence that in mice there may be an intra-ovarian autoregulatory mechanism which controls the rate of development of medium sized follicles, thereby ensuring a constant supply of antral follicles.

We thank the M.R.C. and Wellcome Trust for financial support, and A. Jones and R. Rees for their excellent technical assistance. D.M.G.H. was an M.R.C. Scholar.

\section{References}

Arai, H. (1920) On the postnatal development of the ovary (albino rat), with especial reference to the number of ova. Am. J. Anat. 27, 405-462.

Cattanach, B.M., Iddon, C.A., Charlton, H.M., Chiappa, S.A. \& Fink, G. (1977) Gonadotrophin releasing hormone deficiency in a mutant mouse with hypogonadism. Nature, Lond. 269, 338-340.

diZerega, G.S. \& Hodgen, G.D. (1980) The primate ovarian cycle: suppression of human menopausal gonadotrophin-induced follicle growth in the presence of the dominant follicle. J. clin. Endocr. Metab. 50, 819-825.

Faddy, M.J. (1976) A note on the general time-dependent stochastic compartment model. Biometrics 32, $443-448$.

Faddy, M.J., Jones, E.C. \& Edwards, R.G. (1976) An analytical model for ovarian follicle dynamics. J. exp. Zool. 197, 173-186.

Faddy, M.J., Gosden, R.G. \& Edwards, R.G. (1983) Ovarian follicle dynamics in mice: a comparative study of three inbred strains and an Fl hybrid. $J$. Endocr. 96, 23-33.

Halpin, D.M.G. (1983) Aspects of ovarian function in the mouse. D. Phil. thesis, University of Oxford.

Halpin, D.M.G., Jones, A., Fink, G. \& Charlton, H.M. (1986) Postnatal ovarian follicle development in hypogonadal (hpg) and normal mice, and associated changes in the hypothalamic-pituitary ovarian axis. J. Reprod. Fert. 77, 287-296.

Hirshfield, A.N. \& Midgley, A.R. (1978) The role of FSH in the selection of large follices in the rat. Biol. Reprod. 19,606-611.

Jones, E.C. \& Krohn, P.L. (1961a) The relationships between age, the number of oocytes and fertility in virgin and multiparous mice. J. Endocr. 21, 469-495.

Jones, E.C. \& Krohn, P.L. (1961b) The effect of hypo- physectomy on age changes in the ovaries of mice. $J$. Endocr. 21, 497-509.

Lintern-Moore, S. (1977) Initiation of follicle growth in the infant mouse ovary by exogenous gonadotrophin. Biol. Reprod. 17, 635-639.

Mandl, A.M. \& Zuckerman, S. (1952) Cyclical changes in the number of medium and large follicles in the adult rat ovary. J. Endocr. 8, 341-346.

Nakano, R., Mizumo, T., Katayama, K. \& Tojo, S. (1975) Growth of ovarian follicles in the rat in the absence of gonadotrophins. J. Reprod. Fert. 45, 545-546.

Ojeda, S.R., Andrews, W.W., Advis, J.P. \& Smith White, S. (1980) Recent advances in the endocrinology of puberty. Endocr. Rev. 1, 228-257.

Pedersen, T. (1969) Follicle growth in the immature mouse ovary. Acta endocr., Copenh. 64, 117-132.

Pedersen, T. (1972) Follicle growth in the mouse ovary. In Oogenesis, Ch. 19, pp. 361-376. Eds J. D. Biggers \& A. W. Schuetz. University Park Press, Baltimore.

Pedersen, T. \& Peters, H. (1968) Proposal for a classification of oocytes and follicles in the mouse ovary. $J$. Reprod. Fert. 17, 555-557.

Pedersen, T. \& Peters, H. (1971) Follicle growth and cell dynamics in the mouse ovary during pregnancy. Fert. Steril. 22, 42-52.

Peters H., Byskov, A.G., Lintern-Moore, S., Faber, M. \& Anderson, M. (1973) The effects of gonadotrophins on follicle growth initiation in the neonatal mouse ovary. J. Reprod. Fert. 35, 139-141.

Richards, J.S. (1980) Maturation of ovarian follicles: actions and interactions of pituitary and ovarian hormones on follicle cell differentiation. Physiol. Rev. 60, 5l-89.

Zuckerman, S. (1951) The number of oocytes in the mature ovary. Recent Prog. Horm. Res. 6, 63-109. 